\title{
Autogestión hospitalaria en red II: El método
}

\author{
MARCOS VERGARA $^{(1)}$ y LIGIA BISAMA ${ }^{(2)}$
}

El artículo se refiere al Método desarrollado por la Escuela de Salud Pública (ESP) de la Facultad de Medicina de la Universidad de Chile, utilizado en un programa de acompañamiento para la autogestión hospitalaria en red. El Método persigue facilitar la construcción de tres competencias organizacionales esenciales que deben tener los hospitales públicos para enfrentar el desafío de la autogestión. El Método produce un proceso de aprendizaje para directivos en torno a un proyecto de intervención que se trabaja en grupo y con acompañamiento técnico especializado por parte de la ESP. El propósito del trabajo en grupo es conseguir que éste desarrolle las competencias de equipo que serán necesarias para abordar las competencias esenciales en su hospital.

\section{ANTECEDENTES}

El Programa de Acompañamiento para Hospitales Autogestionados al cual se refiere este artículo, tiene su origen en una iniciativa de la Subsecretaría de Redes del Ministerio de Salud de Chile, impulsada el año 2009 para apoyar a los hospitales públicos que serían certificados el año 2010 como "autogestionados", conforme a la Ley de Autoridad Sanitaria. Para ello, la Subsecretaría consideró un diagnóstico previo de necesidades de los hospitales y las propuestas de distintos oferentes. En lo que respecta a la Escuela de Salud Pública de la Facultad de Medicina de la Universidad de Chile, el Programa consistió en dos experiencias simultáneas de intervención, cada una con directivos de cuatro hospitales de la zona norte y centro sur del país.

En el contexto de la Reforma de Salud en Chile $y$, en particular, del desafío que enfrentan los hospitales llamados a responder a una Política Pública con Enfoque de Derechos ${ }^{1}$, el Programa ESP se propuso vincular, mediante una experiencia de investigación-acción desarrollada por equipos directivos, los ámbitos estratégicos y operativos que conducen a la autogestión hospitalaria.

\section{MARCO TEÓRICO: COMPETENCIAS ORGANIZACIONALES ESENCIALES Y COMPETENCIAS DE EQUIPO}

El propósito de una estrategia organizacional es crear valor, lo cual requiere que toda la organización se involucre en su despliegue. Esto supone que la estrategia ha sido formalmente declarada, que es conocida y visible para todos ${ }^{2}$ y que, además, se desarrolla en condiciones organizacionales que permiten que se devele y surja de la práctica ${ }^{3}$, en un ciclo permanente de aprendizaje.

El valor de las organizaciones se relaciona, entre otras cosas, con los distintos tipos de beneficios que reportan a sus grupos de interés ${ }^{4}$ y con su sostenibilidad en el largo plazo. Para ello, la estrategia debe expresar la forma en que la organización aportará valor a sus grupos de interesados, considerando las tensiones que le imponen sus expectativas a veces en conflicto, el contexto legal y socio-político en que opera y su proyección económico-financiera de largo plazo.

Este hecho conduce a la necesidad de entender muy bien cómo una organización genera valor y cómo es que se producen los aprendizajes que lo hacen posible.

Las opciones estratégicas generan en el tiem-

\footnotetext{
(1) Escuela de Salud Pública. Facultad de Medicina. Universidad de Chile. Independencia 939. Santiago. Chile. mvergara@med.uchile.cl

(2) Emerge Consultores. Santiago. Chile.
} 
po ciertas competencias organizacionales que Hamel y Prahalad ${ }^{5}$ denominaron esenciales, porque contienen y despliegan el aprendizaje colectivo que permite a la organización acceder al mercado potencial, lograr una percepción de beneficios por parte del consumidor y ser difícil de imitar.

Estas competencias esenciales de una organización, según Le Boterf ${ }^{6}$, suponen la contribución, cooperación y sinergia entre varias competencias de quienes forman parte de ésta. Una competencia individual, es una construcción que realiza una persona como resultado de una combinación pertinente de varios recursos: el equipamiento que tiene incorporado (conocimientos, saber hacer, aptitudes, experiencias) y el equipamiento de su entorno (redes de relaciones, instrumentos, bancos de datos, etc.). Es su patrimonio, más o menos desarrollado, parcial o plenamente utilizado. El conocimiento combinatorio es la meta-competencia para construir competencias requeridas. Una persona competente será quien sabe actuar de manera pertinente en un contexto particular, eligiendo y movilizando un equipamiento doble de recursos: personales y de redes. Actuar con competencia implica saber, querer y poder y estos tres puntos deben ser abordados por las organizaciones que pretenden desarrollar competencias individuales y colectivas.

Los equipos de trabajo son un medio para obtener competencias colectivas. Para los hermanos Dyer ${ }^{7}$, las competencias de equipo son uno de los cuatro factores que deben comprenderse y manejarse para lograr un desempeño superior de equipos. Describen 11 competencias que caracterizan a los equipos de alto desempeño; 5 se refieren a la tarea (establecer metas, asignar responsabilidades sobre ellas, tomar decisiones, definir estándares de alto desempeño, realizar reuniones efectivas); otras 5 se refieren a la relación (crear confianza y apoyo; comunicarse en forma abierta, compartir información, expresar sentimientos y retroalimentar; manejar conflictos; evidenciar respeto y colaboración; asumir riesgos). La última, es una meta-

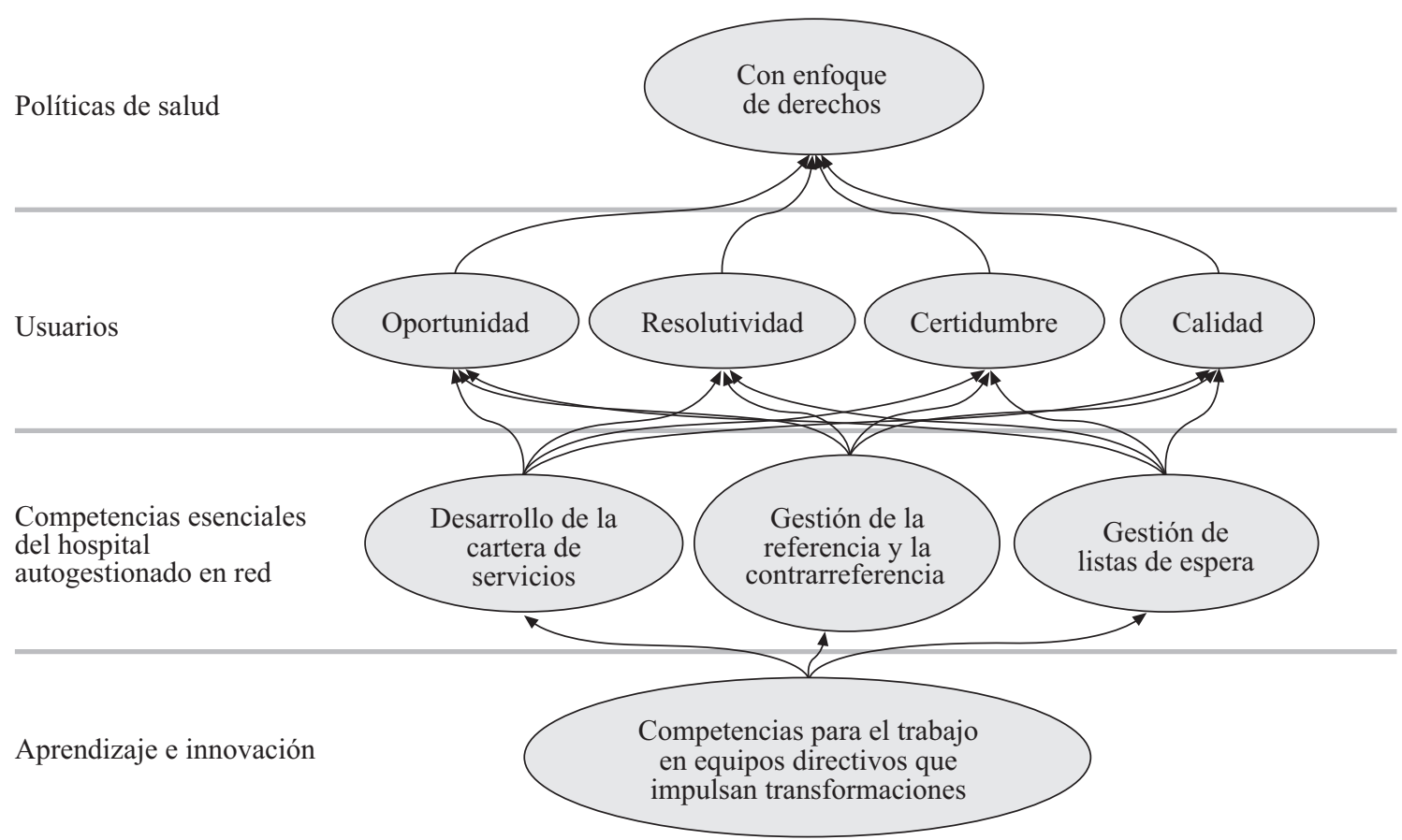

Figura 1. Mapa estratégico de un hospital autogestionado en red. 


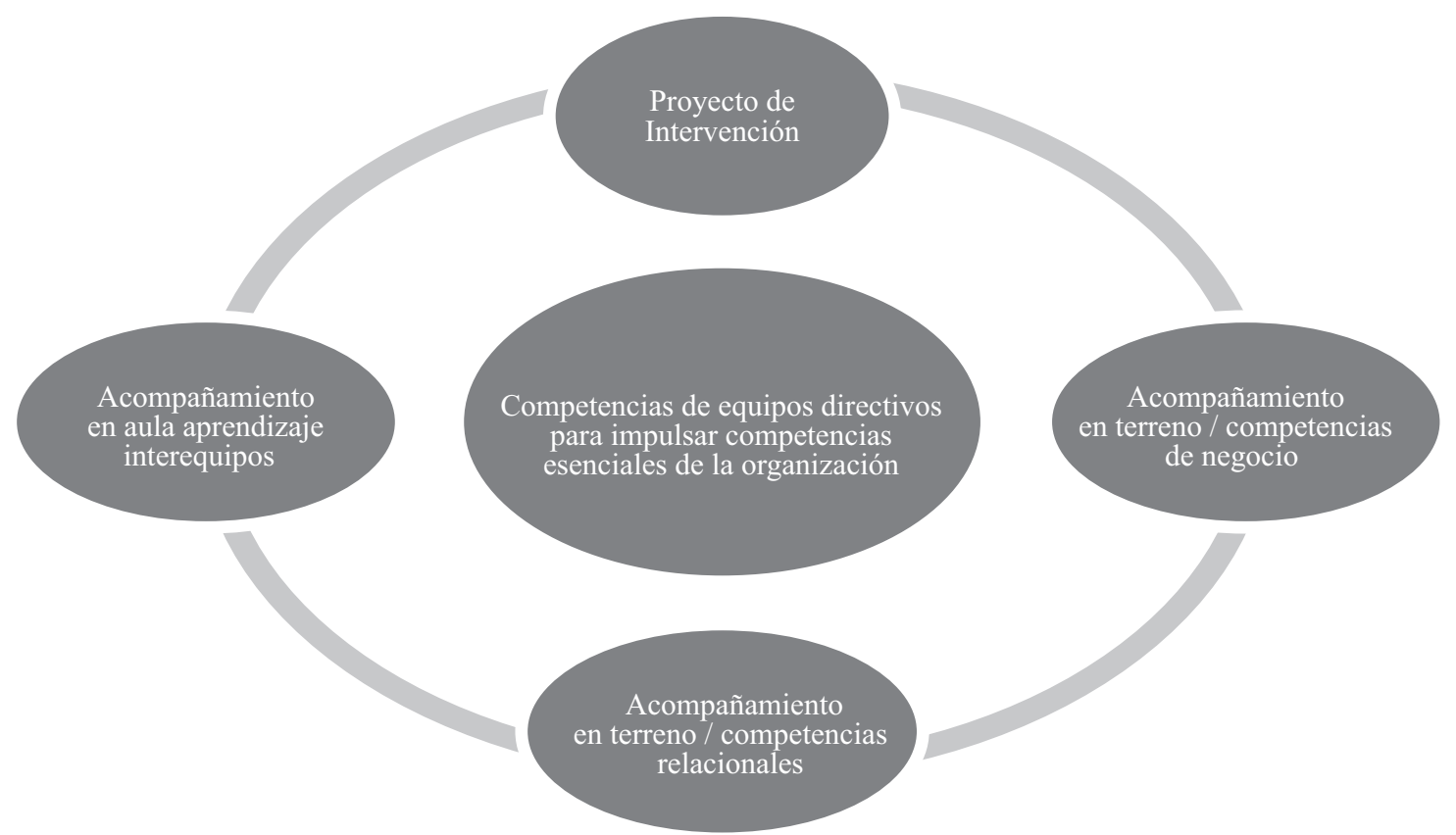

Figura 2

competencia que se refiere a la formación de equipo, es decir la capacidad del equipo para equilibrar sus procesos de tarea y relación y autodiagnosticar su funcionamiento.

Los otros tres factores para el alto desempeño de un equipo son: a) contexto: necesidad organizacional del equipo, tipo de equipo y estructura, sistemas y cultura que lo soportan; b) composición: tamaño, habilidades, experiencia y motivación requeridas y; c) cambio: capacidad del equipo para vigilar su desempeño y cambiar.

Este último factor, cambio, es equivalente al concepto aprendizaje en equipo desarrollado por Senge, quien lo describe en primer lugar como una de las disciplinas que conduce al aprendizaje organizacional. Se diferencia de la capacidad de formar equipo porque "inspira cambios más fundamentales, con una aplicación duradera que se extenderá por la organización"s. Según el autor, el aprendizaje organizacional ocurre cuando existe la necesidad de resolver un problema o de crear algo nuevo, y la rapidez del aprendizaje es mayor, cuando el equipo cuenta con un moderador. El cambio es posible porque en el equipo se ha producido aprendizaje, es decir, se ha obtenido retroalimentación del comportamiento individual, grupal $u$ organizacional y se han tomado decisiones para un desempeño superior. Argyris ${ }^{9}$ introdujo el concepto de aprendizaje de primer orden para referirse al aprendizaje adaptativo o simple; es decir aquel que utilizamos para hacer mejoras. Lo distingue del aprendizaje de segundo orden, porque este último modifica los modelos mentales de quienes están involucrados en él, abriendo otras posibilidades, generando otras estrategias y considerando nuevas reglas para decidir en forma diferente y tomar nuevos cursos de acción ${ }^{10}$.

Considerando lo anterior, se podría afirmar que un equipo que aspira a producir cambios en la organización, no sólo debe manejar competentemente sus propios procesos de tarea y relación, sino que además tener la capacidad de aprender en equipo, es decir, de revisar los modelos mentales con que opera y también aquellos supuestos que rigen el comportamiento de los miembros de la organización en aquellos 
aspectos que son el objetivo de su misión.

Los indicadores de competencia colectiva en una unidad, proceso o proyecto son, según Le Boterf: a) representaciones compartidas, b) comunicación eficaz, c) cooperación eficiente entre los miembros del equipo y, d) saber aprender colectivamente de la experiencia. En esta descripción Le Boterf incorpora tanto la capacidad de un equipo de constituirse como tal, como su capacidad de aprendizaje, es decir, de producir cambio.

Las condiciones favorables para que aparezca las competencias colectivas son, según este autor: i) cooperación entre las competencias (ej. lenguaje común, complementariedad, relación de ayuda), ii) organización que facilite la sinergia entre éstas, iii) asegurar el tratamiento de las interfases, iv) crear y cuidar las relaciones de solidaridad y de buena convivencia, v) instituir procesos de aprendizaje y, vi) dirección apropiada.

Entre los métodos de aprendizaje para el desarrollo de competencias se pueden mencionar la investigación-acción y el coaching.

La aplicación de la investigación-acción al ámbito organizacional ha sido descrita "como un proceso emergente de indagación en el que se aplican conocimientos de las ciencias del comportamiento, que se integran con el saber existente sobre las organizaciones para resolver problemas y desafíos reales y concretos. Se caracteriza por ocuparse simultáneamente de facilitar y gestionar cambios en las organizaciones, desarrollar competencias de autoayuda entre los miembros de la organización y aportar al conocimiento científico. También se distingue por ser un proceso evolutivo que se aborda con un espíritu de colaboración y co-indagación" (Shani y Pasmore, 1985) ${ }^{11}$.

Por su parte, Mario Morales ${ }^{12}$ define el coaching como "un proceso especial y sistemático de relación de ayuda que consiste en facilitar que una persona o equipo (sistema cliente) aprenda por sí mismo, ya sea a resolver sus problemas, a enfrentar desafíos futuros, a mejorar resultados o a desarrollar nuevas competencias, tanto en el plano laboral como personal. Se basa en un sentimiento de confianza en el potencial de crecimiento del sistema cliente, para aprender y desarrollarse y que es puesto a prueba durante el proceso de coaching. Se realiza a través de un proceso comunicacional y de generación de un clima de confianza y respeto que facilita la autoexploración, autorreflexión, autocomprensión y el establecimiento de metas de un plan de mejoramiento o desarrollo".

Existen diversos modelos de coaching cuyas raíces provienen de la filosofía, la teoría de sistemas, la biología y la psicología, por ej. coaching ontológico ${ }^{13}$; Modelo $\mathrm{ICI}^{14}$; o coaching basado en el $\mathrm{ARO}^{15}$, cuyo foco de interés puede ser el desarrollo de una persona, o el desarrollo de competencias de personas y equipos en un contexto organizacional para beneficio recíproco.

\section{ENFOQUE TEÓRICO PARA LA FORMULACIÓN DEL MÉTODO}

En el documento de los autores citado al inicio de este artículo, se concluyó que el éxito de la autogestión hospitalaria en red requiere al menos del desarrollo de tres competencias esenciales en los hospitales:

- Desarrollo de la cartera de servicios.

- Gestión de la referencia y la contrarreferencia.

- Gestión de las listas de espera.

El desarrollo de tales competencias organizacionales esenciales requiere que se produzca un proceso de aprendizaje e innovación en los hospitales que lo haga posible. El enfoque teórico adoptado plantea que uno de los aprendizajes indispensables son las competencias de los equipos directivos que permitirán impulsar el desarrollo de dichas competencias esenciales en sus hospitales.

Así, cuando los directivos hayan desarrollado competencias para actuar en equipo con el propósito de generar el cambio organizacional que implican las tres competencias esenciales, los procesos organizacionales internos involucrados, se expresarán en resolutividad, oportunidad, certidumbre (acerca de cómo, cuándo y dónde se realizará la atención) y calidad de servicios para los usuarios. La satisfacción y confianza 
de los usuarios en el sistema permitirá, a su vez, alcanzar el objetivo estratégico de la política pública con enfoque de derechos, cuidando y/o agregando valor para todos los actores involucrados.

Se tomó la decisión de iniciar el proceso de aprendizaje para el desarrollo de las tres competencias esenciales mediante el desarrollo de equipos directivos por las siguientes razones:

Tanto las competencias de los equipos de trabajo que se refieren a comportamiento de tarea y de relación, así como la capacidad para auto-diagnosticar y manejar ambos procesos pueden ser descritas. Esta aproximación ofrece posibilidad de especificar qué comportamientos de tarea y relación de los equipos directivos apuntan a la construcción de competencias esenciales de los hospitales. También permiten una selección, definición y evaluación más acotada de indicadores de competencia colectiva como los señalados por Le Boterf.

Por otra parte, son los equipos directivos, quienes desplegando sus competencias, están en mejor posición mediante sus decisiones, para facilitar el desarrollo de las condiciones organizacionales que favorecen la aparición de las competencias colectivas.

Finalmente, a partir del marco teórico, se entendió que las competencias de los equipos directivos de los hospitales, son una construcción del equipo a partir de los recursos individuales que sus integrantes poseen y de su acceso al equipamiento del entorno. El aprendizaje en equipo es la meta-competencia que requerirán para construir las competencias esenciales de sus hospitales.

\section{MÉTODO}

El método consiste en desarrollar competencias de equipos directivos que impulsen a su vez la construcción de las tres competencias esenciales en los hospitales públicos. Las competencias organizacionales esenciales se abordan a través de proyectos de intervención formulados e implementados con un enfoque de "investigación-acción" por equipos conformados por directivos de los hospitales que participan en el Programa.

Las competencias organizacionales esenciales se formulan como objetivos generales comunes a todos los proyectos. Los objetivos específicos de cada proyecto, sus indicadores y líneas de base responden a las necesidades de cada hospital. Cada equipo define metas y un conjunto de actividades orientadas a lograrlas las cuales

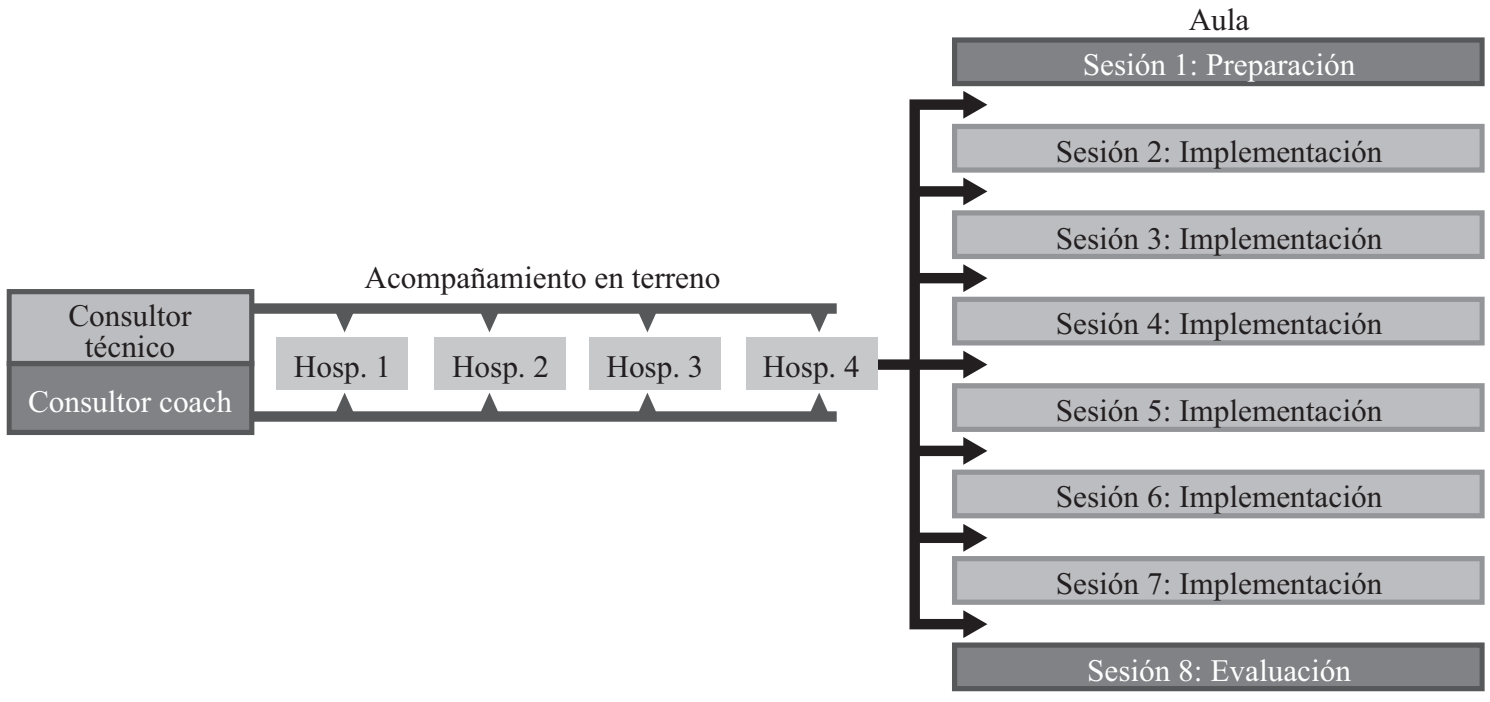

Figura 3 
son evaluadas al término del Programa y más allá de éste, con un horizonte de un año. Los proyectos abordados con este método, reflejan el vínculo entre los ámbitos estratégicos y operativos de la gestión hospitalaria.

Tanto las competencias de equipo consideradas como necesarias para llevar a la práctica el proyecto de intervención, como los indicadores que dan cuenta de su nivel de desarrollo, se describen en un documento. Respecto de la tarea, esta descripción es usada para mantener tanto la atención en el propósito del proyecto de intervención (construcción de las competencias esenciales), como en los aspectos operativos involucrados en su implementación. Respecto de la relación, los comportamientos son descritos de tal modo que guíen a los participantes a relacionarse entre sí, y con otros actores relevantes para su proyecto, de acuerdo a los criterios que caracterizan a un equipo de trabajo. Por último, la descripción de competencias también se refiere a los comportamientos que indican aprendizaje en equipo.

El instrumento es usado por los equipos directivos para autoevaluarse antes, durante y después del proceso de implementación de su proyecto.

Las competencias de los equipos directivos se desarrollan con el apoyo de dos tipos de acompañamiento en terreno en los respectivos hospitales. Uno de ellos toma la forma de consultoría para los aspectos técnicos involucrados en el desarrollo de las tres competencias esenciales del "negocio" (la tarea); mientras que el otro consiste en un programa de coaching para el aprendizaje de competencias relacionales de equipo que permitirán impulsar con éxito el proyecto (la relación). Tanto los consultores como los coach se ocupan también de los procesos de aprendizaje en equipo.

Además, el método considera un espacio para la reflexión y aprendizaje entre equipos directivos de los diferentes hospitales. A este espacio, se le denomina "aula" y pone énfasis en la perspectiva estratégica de los proyectos y en el desarrollo de competencias de aprendizaje en equipo.

El acompañamiento en aula y en terreno se realiza por una dupla constituida por un consultor (experto en gestión en salud pública) y un coach (psicólogo experto en desarrollo de equipos).

En el Programa de Acompañamiento para la Autogestión Hospitalaria realizado con cuatro hospitales de la zona norte y cuatro de la zona centro sur del país, la aplicación del Método descrito tomó la forma que indica la Figura 3.

\section{CONCLUSIONES}

El Método adoptado permite poner en marcha un proceso de aprendizaje para que un conjunto de directivos desarrollen competencias de equipo en torno a un proyecto orientado a desarrollar tres competencias esenciales de su hospital.

Estas tres competencias esenciales de los hospitales públicos -gestión de la cartera de servicios, de la referencia y contra-referencia y de las listas de espera- tienen el carácter de esenciales porque son imprescindibles para satisfacer una Política Pública con Enfoque de Derechos.

El Método se aboca al desarrollo de competencias de equipos directivos porque son competencias colectivas que ofrecen un espacio de aprendizaje acotado y porque los directivos tienen el poder para producir impacto organizacional en la medida que efectivamente actúen como un equipo competente.

El aspecto distintivo del Método radica en que aborda el desafío de la autogestión hospitalaria articulando competencias organizacionales esenciales y competencias de equipos directivos. Además, a través de los proyectos, permite dar visibilidad y consistencia a la gestión estratégica y operativa del hospital.

\section{REFERENCIAS}

1. VERGARA M. Y BISAMA L. Auto-gestión hospitalaria: competencias esenciales para la gestión. Artículo en revisión para publicación, marzo 2010.

2. KAPLAN, R.; \& NORTON D. Cómo utilizar el cuadro de mando integral para implementar y gestionar su Estrategia. Gestión 2000, S.A., Barcelona, 2001.

3. MINTZBERG, H. Minzberg y la Dirección. Ediciones Diaz de Santos, S.A., Madrid, España, 1991. 
4. KRICK, T.; FORSTATER, M.; MONOGHAN, P. \& SILLANPÄ̈̈, M. De las palabras a la acción. El compromiso con los stakeholders. Manual para la práctica de las relaciones con los grupos de interés. Copyright 2005 Accountability United Nations Enviroment Programme Stakeholders Research Associates Canada. Ink. Primera versión castellana, enero 2006. Disponible en internet: www.accountability.org.uk, www.stakeholdersresearch.com, www.uneptie.org (pág.10).

5. PRAHALAD, G. \& Hamel, G. The core competence of the corporation. Harvard Business Review, mayojunio, 1990.

6. LE BOTERF, G. Ingeniería de las competencias. Ediciones Gestión 2000 S.A.; Barcelona, febrero 2001.

7. DYER, W.; DYER G. \& DYER J. Equipos de trabajo de alto desempeño. Estrategias infalibles para perfeccionarlos. Copyright 2007. Grupo Editorial Patria; México 2008.

8. SENGE, P. La quinta disciplina en la práctica. Editorial Granica, Buenos Aires, Argentina, 1995 (pág. 368).
9. ARGYRIS, C; PUTMAN, R Y SMITH, D, Action Science, Jossey-Bass, 1985.

10. O'CONNOR J.; MC DERMONTT, I. Introducción al pensamiento sistémico. Recursos esenciales para la creatividad y resolución de problemas. Editorial Urano, 1997.

11. SHANI Y PASMORE. 1985. Doing action research; p. 439. Accesible en http://www.sagepub.com/upmdata/9826 039584ch1and2.pdf

12. MORALES, M. Fundamentación y análisis de los conceptos básicos y del coaching como relación de ayuda. TOP Consultores S.A. Santiago. Chile. 2002.

13. ECHEVERRÍA, R. Ontología del Lenguaje. RIL Editores. Santiago. Chile. 2008.

14. ANWANDTER, P. Introducción al Coaching integral. Ril editores, Santiago, Chile 2008 (pág. 33).

15. ACUÑA, E. \& SANFUENTES, M. (editores) Coaching. Análisis del rol organizacional. Facultad de Economía y Negocios de la Universidad de Chile. Editorial Universitaria. Santiago de Chile. Mayo 2009 (Pág. 15). 